\title{
On the relationship between the geometrical control of scintillation indices and the data detrending problems observed at high latitudes
}

\author{
Biagio Forte \\ Centre for Atmospheric Research, University of Nova Gorica, Slovenia
}

\begin{abstract}
The experimental estimate of radio waves scintillation, caused by plasma density irregularities in the ionosphere, is usually attempted by means of scintillation indices which are essentially standard deviations of stochasticly fluctuating parts of the received radio wave intensity and phase. At high latitudes, provided that the propagation problem may be modelled by means of the weak scattering theory, the typical scintillation indices $S_{4}$ and $\sigma_{\phi}$ depend on a geometrical factor which introduces some amplifications on their values. Scintillation indices $S_{4}$ and $\sigma_{\phi}$ measured at auroral latitudes are estimated by means of different boundary detrending conditions and the geometrical effect on those detrending conditions is investigated. In the case of the polar Low Earth Orbiting (LEO) satellite links considered here, high phase with low intensity scintillation events do not seem to be related to geometrical effects only, but rather to misleading data detrending.
\end{abstract}

Key words ionospheric scintillation - GPS scintillation monitors - scintillation indices

\section{Introduction}

The amount of scintillation activity present in radio waves propagating through the upper atmosphere is estimated by means of scintillation indices which measure the stochastic fluctuating components of these electromagnetic radiations. The scintillation activity is assessed on both intensity and phase of radio signals received on the ground, after propagating through

Mailing address: Dr. Biagio Forte, Centre for Atmospheric Research, University of Nova Gorica, Vipavska 13, PO Box 301, SI-5001 Nova Gorica, Slovenia; e-mail: biagio.forte@p-ng.si drifting irregularities of the electron density spatial distribution in the ionosphere.

The scintillation index, used overall for estimating the fluctuations induced by drifting plasma density irregularities on the signals intensity, is the index $S_{4}$ (Briggs and Parkin, 1963), i.e. the intensity standard deviation normalised to the average received intensity and is given by

$$
S_{4}^{2}=\frac{\left\langle I^{2}\right\rangle-\langle I\rangle^{2}}{\langle I\rangle^{2}}
$$

where $I$ is the received intensity and the $\langle$.$\rangle op-$ erator denotes an ensemble average.

The phase scintillation index $\sigma_{\phi}$ is usually defined as the standard deviation of the received phase. Of course, these standard deviations are aimed to estimate the signal fluctuations induced by radio wave scintillations. Thus, the signal components subject to fluctuations due to ionos- 
pheric scintillation (i.e., caused by drifting electron density irregularity structures in the ionosphere) need to be identified and separated from other signal components. This is usually accomplished by means of a detrending process applied to the intensity and phase of the received radio signal, filtering out those signal components not pertaining to the scintillation mechanism. The detrending process usually takes place in the frequency domain and is characterised by a low frequency edge below which signal fluctuations and trends are assumed to be not related to ionospheric scintillation. The choice of such a cut-off frequency depends on the geometry of the propagation problem considered. The key point is to choose the cut-off frequency low enough to catch the bulk of intensity scintillation, leaving $S_{4}$ unmodified for all the experimental conditions, determined by the signal wavelength and by the ionospheric relative drift. The cut-off frequency choice clearly depends on the experimental features implying that a general value does not exist. For instance, in the case of the past Wideband experiment based on a polar low Earth orbiting satellite transmitting several radio beacon wavelengths, such a value was fixed to be $0.1 \mathrm{~Hz}$ (Fremouw et al., 1978).

This has been the cut-off frequency value used overall, in both high and low latitude experiments dealing with generic beacon satellites, although not always LEO. In the last decade, the rising interest in assessing the impact of ionospheric scintillation on satellite based navigation systems has led to the development of GPS scintillation monitors allowing for the retrieval of the scintillation information from all the satellites on track (Van Dierendonck et al., 1993; Beach and Kintner, 1999). The detrending process used in these monitors relies on a fixed low frequency cut-off edge equal to $0.1 \mathrm{~Hz}$, as in the case of beacon satellites based experiments.

It has been a common and accepted picture that GPS scintillation monitors detect high phase scintillation in the presence of low intensity scintillation at high latitudes, implying that GPS equipment is subject to disturbances in the phase rather than on the intensity of the signals, irrespective of how this may impact the functioning of GPS receivers (Doherty et al., 2000; Pi et al., 2001; Aquino et al., 2007).
A possible explanation of "phase without amplitude» scintillation events routinely observed by GPS scintillation monitors at high latitudes is based on erroneous data detrending, due to a fixed cut-off frequency $(0.1 \mathrm{~Hz})$ not appropriate to the propagation conditions which may occur at high latitudes for GPS signals (Forte and Radicella, 2002). Moreover, there seems to be no clear evidence for a geometrical control of the scintillation indices as measured on GPS links (Forte and Radicella, 2004), similar to what is observed with polar LEO beacon satellites which leads to enhancements of the phase scintillation index whenever the ray path lies within an $L$-shell (Fremouw, 1980).

Here, some scintillation events measured by means of polar LEO beacon satellites are analysed to explain how the classical phase scintillation index $\sigma_{\phi}$ may introduce some troubles in the estimate of the phase scintillation activity on a given satellite link. This takes into account possible geometrical effects on $\sigma_{\phi}$ and an alternate index for the assessment of phase fluctuations induced by drifting electron density irregularities at ionospheric heights (Forte, 2005).

\section{Experimental data}

The experimental data used here are based on radio signals coherently transmitted from Tsykada beacon satellites at two frequencies: $150 \mathrm{MHz}$ and $400 \mathrm{MHz}$. These radio signals are received at nearly aligned ground stations which are used overall for tomographic ionospheric reconstruction at European auroral latitudes (fig. 1). The intensity and phase of the received radio signals are sampled at a $50 \mathrm{~Hz}$ rate making it possible to estimate the scintillation activity experienced while traversing drifting electron density irregularities in the ionosphere. No elevation mask angle is applied to the data, so that border effects at the beginning and end of the time series shown hereafter are associated mainly with low elevation angle phenomena.

A detrending process is applied to the raw data to select only those stochastic fluctuations induced by drifting plasma density irregularities on both intensity and phase of the signals received on the ground. The detrending process is 
essentially a high-pass filter designed in the frequency domain and characterised by a fixed cutoff low frequency edge. All the fluctuations corresponding to frequencies below this cut-off are

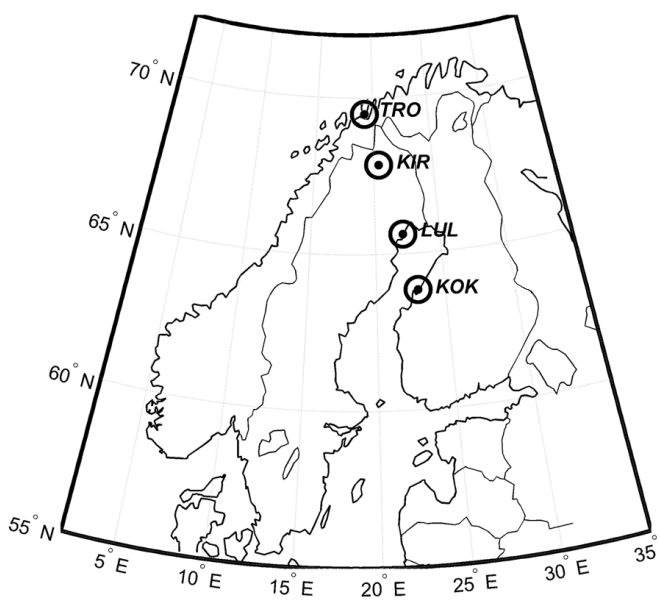

Fig. 1. The ground stations used for recording Tsykada signals: Tromso (TRO), Kiruna (KIR), Lulea (LUL), and Kokkola (KOK).
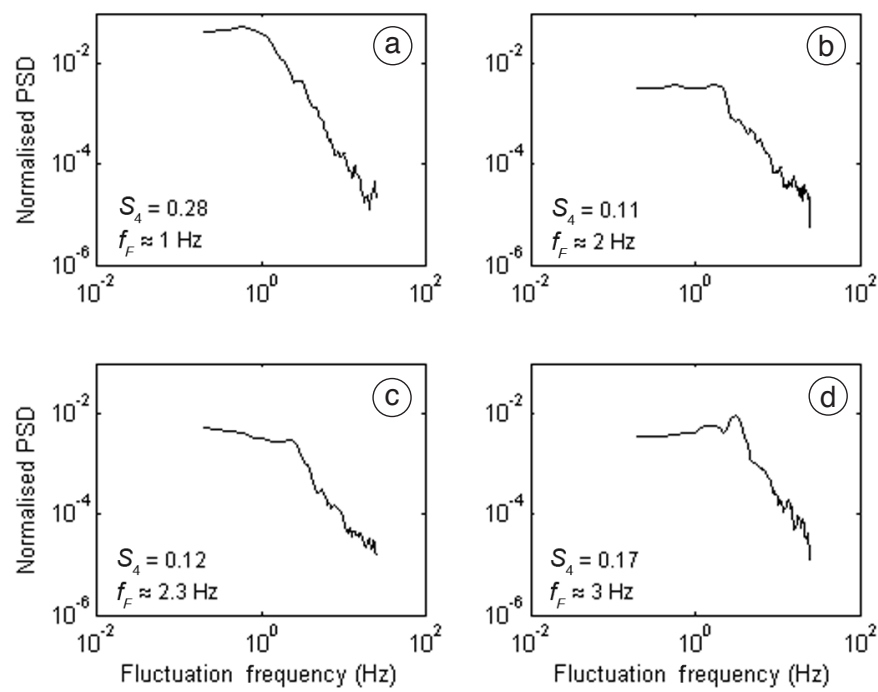

ruled out from the calculation of the scintillation indices, as they are considered not pertaining to ionospheric scintillation. The scintillation indices $S_{4}$ and $\sigma_{\phi}$ are calculated for three different values of the cut-off low frequency edge: $0.1 \mathrm{~Hz}$, $0.3 \mathrm{~Hz}$, and $0.5 \mathrm{~Hz}$. The values assumed for the low frequency limit in the detrending process are consistent with the typical values for the Fresnel fluctuation frequencies relative to the geometry of satellites links considered.

The Fresnel frequency is given by

$$
f_{F}=\frac{V^{\mathrm{REL}}}{d_{F}}
$$

where $V^{\mathrm{REL}}$ is the relative drift at ionospheric altitudes composed of the satellite motion and the electron density irregularities drift, while $d_{F}$ is the Fresnel radius. Considering that at high latitudes plasma density irregularity structures may have drift speeds ranging from about 100 $\mathrm{m} / \mathrm{s}$ to about $1 \mathrm{~km} / \mathrm{s}$ (Aarons, 1982), the Fresnel frequencies for the Tsykada satellites links are expected to be around a few $\mathrm{Hz}$.

This is also confirmed by the experimental data. Figure $2 \mathrm{a}-\mathrm{d}$ shows some scintillation events observed at Kiruna (Lat: $67.84^{\circ} \mathrm{N}$, Long: $20.41^{\circ} \mathrm{E}$,

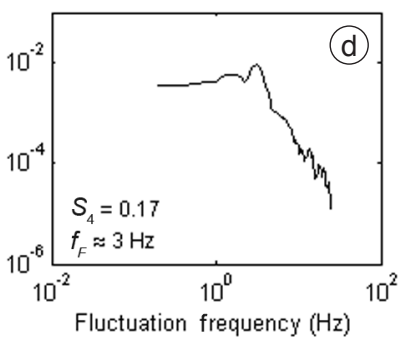

Fig. 2a-d. Intensity power spectral densities normalised to the mean received intensity, as measured for different satellite links. This shows that a Fresnel frequency of a few $\mathrm{Hz}$ may normally occur at auroral latitudes. The measurements are taken during the night of 30 october 2003 from a) Kokkola, b) Kiruna, c) and d) Lulea. 
L: 5.6) during the night of 30 October 2003. The power spectral densities corresponding to $20 \mathrm{~s}$ time intervals show Fresnel frequencies of a few $\mathrm{Hz}$ as indicated, owing to real values for the relative ionospheric drift, for the distance of the receiver to the phase screen approximating the electron density structures, and for the satellite zenith angle. The values chosen for the cut-off frequency in the data detrending process are aimed to show how $S_{4}$ and $\sigma_{\phi}$ estimates modify as the low frequency detrending limit is shifted towards higher fluctuation frequencies, owing to Fresnel frequencies of a few $\mathrm{Hz}$ for the links considered here. The key point is understanding whether large $\sigma_{\phi}$ values may always be explained in terms of geometrically induced enhancements or in terms of misleading data detrending conditions as well (Forte, 2005).

\section{Discussion}

Figure 3 shows scintillation indices measured for the $400 \mathrm{MHz}$ radio signal during a satellite pass over the ground station located in Lulea (Lat: $65.58^{\circ} \mathrm{N}$, Long: $22.17^{\circ} \mathrm{E}, \mathrm{L}: 4.7$ ) on the night of 30 October 2003 starting at 19:30:29 UT. The top left plot shows $S_{4}$ values for the three different low frequency detrending limits: the solid line refers to a cut-off frequency of 0.1 $\mathrm{Hz}$, the dashed line refers to a cut-off frequency of $0.3 \mathrm{~Hz}$, while the dotted line refers to a cut-off frequency of $0.5 \mathrm{~Hz}$. The top right plot shows $\sigma_{\phi}$ values in radians for the same cut-off frequency values. The bottom left plot shows a new parameter proposed for the estimate of phase scintillation, which is given by (Forte, 2005)

$$
S_{\phi}=\sqrt{\left\langle\left(\frac{\partial \phi}{\partial t}\right)^{2}\right\rangle}
$$

where the $\langle$.$\rangle operator denotes an ensemble av-$ erage and $\phi$ is the received and detrended phase. $S_{\phi}$ values are given in $\mathrm{rad} / \mathrm{s}$. The bottom right plot shows the geometrical factor $G$ (in arbitrary units) responsible for amplifications of $\sigma_{\phi}$ values. For axially symmetric irregularity structures $G$ achieves a maximum only at the magnetic zenith, while for sheetlike structures $G$ maximises whenever the propagation vector lies within the plane of the sheet which is also $L$-shell aligned (Rino, 1979). The $G$ factor
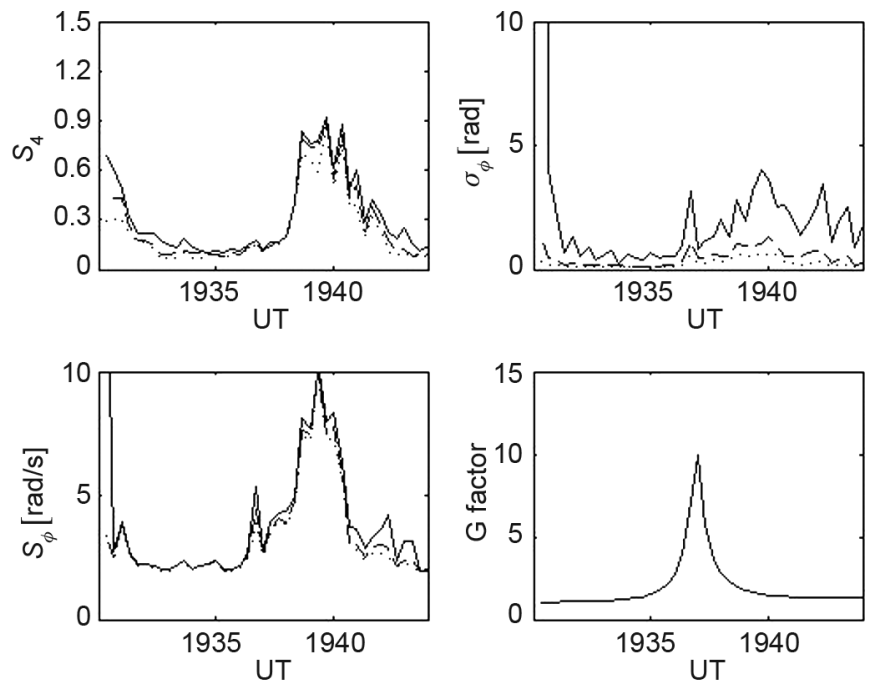

Fig. 3. Scintillation indices recorded during the night of 30 October 2003 from the ground station located in Lulea. The solid lines refer to a detrending cut-off frequency of $0.1 \mathrm{~Hz}$, the dashed lines to $0.3 \mathrm{~Hz}$, and the dotted lines to $0.5 \mathrm{~Hz}$. Also shown the geometrical factor $G$ for this satellite link. 
shows a localised peak for a pierce point latitude between $60^{\circ}$ and $70^{\circ}$ which is associated with a local peak in both $\sigma_{\phi}$ and $S_{\phi}$ occurring slightly after 19:35:00 UT. No similar localised peaks appear in $S_{4}$ which takes low values in the neighbourhood of the localised peak in $G$. This seems to be a typical situation where a radio wave with a ray path aligned with an $L$-shell is weakly scattering on an electron density irregularity structure. For all the events shown here the $G$ factor has been calculated assuming sheetlike electron density irregularities structures, which appear to be typical at auroral latitudes. The axial ratios which define $G$ are those relative to sheetlike irregularities, i.e. 8:8:1 (Rino and Owen, 1980).

The values of $\sigma_{\phi}$ indeed experience an enhancement due to a pure geometrical factor which is perfectly described by means of the weak scattering theory (Rino, 1979). After this peak, an overall increase in the scintillation activity is recorded along the ray path which is not aligned with a magnetic field line anymore. The detrending effect is clearly visible on the scintillation indices: an evident rescaling of $\sigma_{\phi}$ occurs when increasing the value of the low cut-off frequency detrending limit, while such a rescaling is essentially negligible on both $S_{4}$ and $S_{\phi}$. In particular, the rescaling observed on $\sigma_{\phi}$ shows how the detrending effect and the geometrical effect are separate and consistent with each other. The $\sigma_{\phi}$ enhancement caused by purely geometrical reasons is still evident on its rescaled values as well as on the $S_{\phi}$ index. Moreover, rescaled values of $\sigma_{\phi}$ are still tracking the higher scintillation event starting after 19:38:00 UT, in accordance with $S_{4}$ and $S_{\phi}$ behaviour.

A slightly different situation is depicted in fig. 4, which shows scintillation indices measured for the $400 \mathrm{MHz}$ signal during a satellite pass over the ground station located in Kiruna on the night of 30 October 2003, beginning at 23:11:39 UT. High scintillation activity is present on this link as shown by all the three indices. There is a peak in $G$ which appears to be soft, owing to a limited ray path alignement with $L$ shells. The geometrical effect on $\sigma_{\phi}$ is probably taking place, but very difficult to notice, due to the mixing with an overall high scintillation activity present throughout the entire satellite pass.
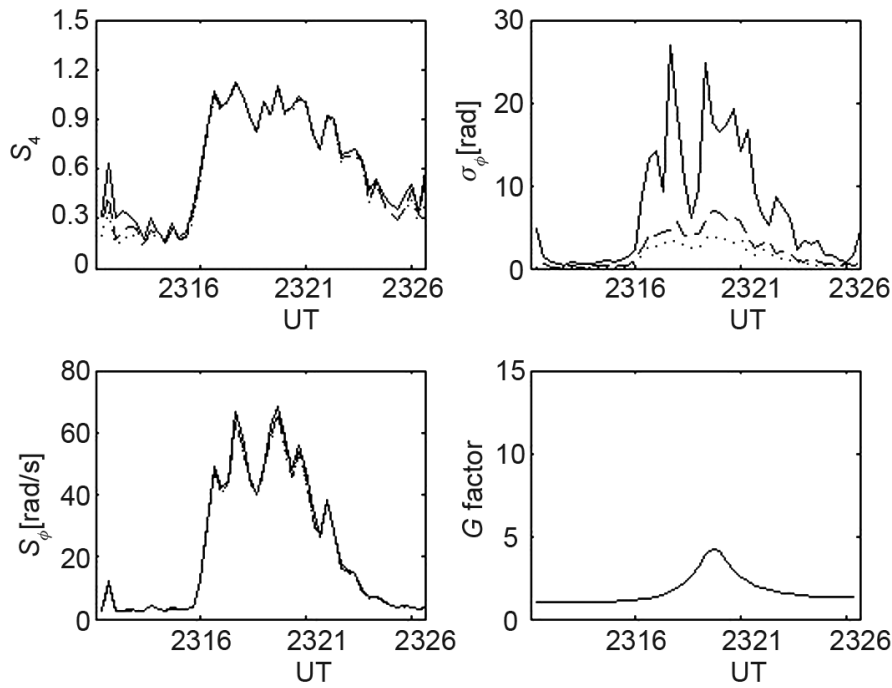

Fig. 4. Scintillation indices recorded during the night of 30 October 2003 from the ground station located in Kiruna. The solid lines refer to a detrending cut-off frequency of $0.1 \mathrm{~Hz}$, the dashed lines to $0.3 \mathrm{~Hz}$, and the dotted lines to $0.5 \mathrm{~Hz}$. Also shown the geometrical factor $G$ for this satellite link. 
On the contrary, the detrending effect is clearly visible: again, an evident rescaling of $\sigma_{\phi}$ values occurs when the low frequency boundary of the detrending process is shifted towards higher fluctuation frequencies. Such a rescaling does not occur for both $S_{4}$ and $S_{\phi}$ which show almost the same values in the three different detrending conditions. Nevertheless, the $\sigma_{\phi}$ values corresponding to cut-off frequencies of $0.3 \mathrm{~Hz}$ (dashed line) and $0.5 \mathrm{~Hz}$ (dotted line) are still tracking the high scintillation event present throughout the link as their behaviour is consistent with both $S_{4}$ and $S_{\phi}$ trends.

Although $S_{4}$ and $S_{\phi}$ values remain unmodified after changing the detrending cut-off frequency, the question is still whether the correct cut-off frequency for $\sigma_{\phi}$ is $0.3 \mathrm{~Hz}$ or $0.5 \mathrm{~Hz}$. This clearly depends on the Fresnel frequency values corresponding to each link geometry. The Fresnel frequency may vary even during a single satellite pass owing to the changing satellite position, to the drift of the irregularity structures along the ray path, and to the distance of the receiver from the phase screen as- sociated with those structures. The difficulties related to the choice of a unique detrending cutoff frequency, suitable for a proper estimate of phase scintillation, make the classical $\sigma_{\phi}$ a problematic parameter (Forte, 2005; Beach, 2006). Although this has been already observed in the case of GPS satellites links at high latitudes and explained in terms of weak scattering results (Forte, 2005; Forte et al., 2004; Beach, 2006), the data presented here show that the detrending problem may be a critical issue also in the case of different satellite links, unless a careful analysis of the propagation geometry and conditions is performed in order to establish the most appropriate cut-off frequency value. For instance, an example of such a careful analysis was provided in the Wideband experiment (Fremouw et al., 1978).

Figure 5 provides the last case study to be considered. Scintillation indices observed on the $400 \mathrm{MHz}$ link are plotted. These indices refer to the satellite pass started at 18:16:31 UT and received from the ground station located in Kiruna. Low scintillation activity is observed on this
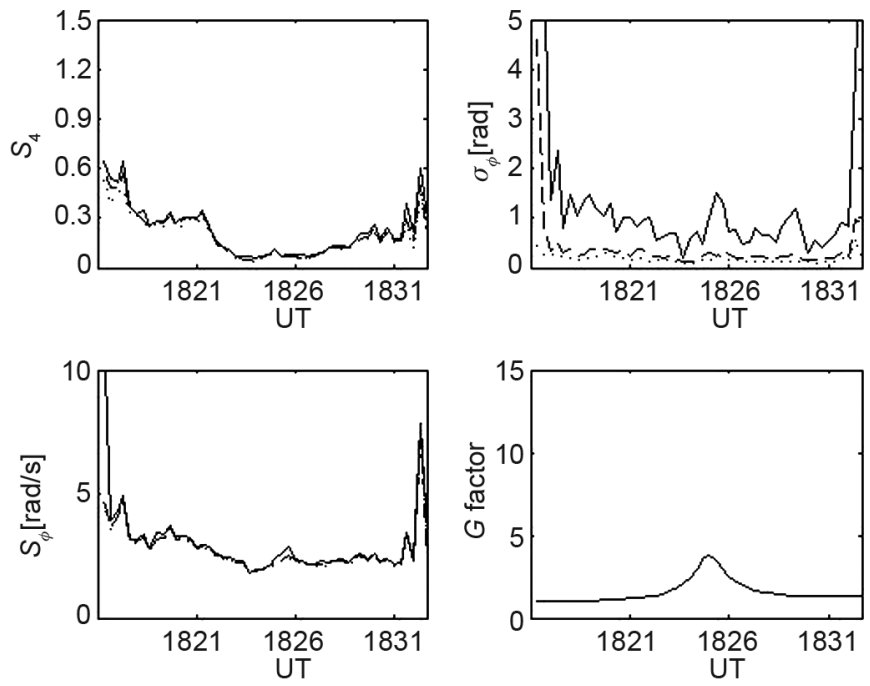

Fig. 5. Scintillation indices recorded during the night of 30 October 2003 from the ground station located in Kiruna (for a time interval different than in fig. 4). The solid lines refer to a detrending cut-off frequency of 0.1 $\mathrm{Hz}$, the dashed lines to $0.3 \mathrm{~Hz}$, and the dotted lines to $0.5 \mathrm{~Hz}$. Also shown the geometrical factor $\mathrm{G}$ for this satellite link. 
satellite pass. Neglecting the initial and final epochs which correspond to low satellite elevation, $S_{4}$ is around 0.3 first and mostly around 0.1 later. $S_{\phi}$ is also mostly around $2 \div 3 \mathrm{rad} / \mathrm{s}$, which correspond to low scintillation values, as may be noticed by comparing the $S_{\phi}$ values appearing in fig. 5 and those in figs. 3 and 4 relative to high scintillation events. The classical phase scintillation index $\sigma_{\phi}$ records values around and in excess of $1 \mathrm{rad}$ for a typical cut-off frequency of $0.1 \mathrm{~Hz}$, while for cut-off frequencies of $0.3 \mathrm{~Hz}$ and 0.5 $\mathrm{Hz}$ there is a significant drop to lower values, more appropriate to low scintillation conditions. Both $S_{4}$ and $S_{\phi}$ maintain almost the same values for all the three different low frequency boundary conditions in the detrending process, as the difference between the corresponding values is negligible.

The three case studies show how $S_{4}$ and $S_{\phi}$ are not affected by misleading data detrending, as they maintain their values unmodified when shifting the detrending cut-off frequency towards even higher fluctuation frequencies. This confirms the intrinsic weakness of $\sigma_{\phi}$ for the estimate of the phase scintillation activity, particularly at high latitudes, and the evident capability of the new $S_{\phi}$ parameter to properly assess the impact of ionospheric scintillation on the phase of a radio wave.

As may be noticed in fig. 5, a less pronounced peak is present in $G$ which corresponds to a peak in $\sigma_{\phi}$, still visible for the $0.3 \mathrm{~Hz}$ and 0.5 $\mathrm{Hz}$ lines. To some extent, such a peak is also reflected in $S_{\phi}$. This proves again that the geometrical effect and the detrending effect are separate and consistent with each other. The geometrical effect only cannot satisfactorily explain the occurrence of high $S_{\phi}$ values with low $S_{4}$ values, not only in the case of GPS satellites links but also in the case of Tsykada satellites links. Even in the case of polar LEO satellites links, it seems that a misleading data detrending may still be a critical issue in the estimate of the classical phase scintillation index $S_{\phi}$.

\section{Conclusions}

The analysis of the scintillation activity recorded on Tsykada satellites links through the estimate of the classical indices $S_{4}$ and $S_{\phi}$, coupled with the new parameter $S_{\phi}$, clarifies the relationship between the geometrical effect which may control $\sigma_{\phi}$ in presence of weak scattering and the detrending effect which may introduce misleading large $\sigma_{\phi}$ values, particularly at high latitudes.

The case studies considered indicate that the geometrical effect and the detrending effect are separate and consistent with each other. Enhancements in $\sigma_{\phi}$ owing to localised peaks in the geometrical factor $G$ remain evident even after the rescaling produced by the increase in the low frequency boundary of the data detrending process. Only the geometrical effect cannot explain the overall occurrence of high $\sigma_{\phi}$ values with low $S_{4}$ values, as this effect is very localised and limited only to those satellite positions making the ray path aligned with an $L$ shell. In case of satellite passes mostly dominated by high scintillation activity, the geometrical effect seems not to be very evident, while the detrending effect on $\sigma_{\phi}$ appears to be indeed very evident.

The geometrical effect also seems to be present on the new parameter $S_{\phi}$ which does not depend on the low frequency boundary conditions, while $S_{4}$ does not show any geometrical or detrending effects.

The $S_{\phi}$ parameter appears to be a more robust indicator for the estimate of radio waves ionospheric phase scintillation, not only in the case of GPS links but also for overall polar LEO satellites links, such as the Tsykada ones.

It should be noticed that the $S_{\phi}$ index is not related to the existing theory on radio wave scintillations, but more tailored to the operational aspects of satellite based navigation systems. Such an index, indeed, is aimed to estimate the signal dynamics caused by ionospheric scintillations which may even lead to signal loss of lock. In this respect, $S_{\phi}$ seems to provide some useful insights which may result of particular interest in designing future satellite navigation based devices.

The events shown here refer to three different satellite passes at auroral latitudes. A larger statistics is nevertheless needed and will be investigated in forthcoming studies to confirm these initial insights. 


\section{Acknowledgements}

The work related to this paper was funded by the Slovenian Research Agency. The author would like to thank the Sodankyla Geophysical Observatory (University of Oulu, Finland) for kindly sharing some of the Tsykada satellites data.

\section{REFERENCES}

AARONS, J. (1982): Global morphology of ionospheric scintillations, Proc. IEEE, 70 (4), 360-378.

Aquino, M., M. Andreotti, A. Dodson and H. StrangeWAYS (2007): On the use of ionospheric scintillation indices as input to receiver tracking models, Adv. Space Res., 40, 426-435.

BEACH, T.L. (2006): Perils of the GPS phase scintillation index, Radio Sci., 41 (5), RS5S31, doi: 10.1029/ 2005RS003356

BEACH, T.L. and P.M. KINTNER (1999): Simultaneous global positioning system observations of equatorial scintillations and total electron content fluctuations, J. Geophys. Res., 104 (A10), 22535-22565.

Briggs, B.H. and I.A. PARKIN (1963): On the variation of radio star and satellite scintillations with zenith angle, J. Atmos. Terr. Phys., 25, 339-365.

DOHERTY, H.P., S.H. Delay, C.E. Valladares and J.A. KLOBUCHAR (2000): Ionospheric scintillation effects in the equatorial and auroral regions, Paper presented at Institute of Navigation GPS 2000, September 2000, Salt Lake City, Utah.

FORTE, B. (2005): Optimum detrending of raw GPS data for scintillation measurements at auroral latitudes, J. Atmos. Sol-Terr. Phys., 67, 1100-1109.
Forte, B. and S.M. RAdicElla (2002): Problems in data treatment for ionospheric scintillation measurements, Radio Sci., 37 (6), 1096, doi: 1029/2001RS002508.

FoRTE, B. and S.M. RADICELLA (2004): Geometrical control of scintillation indices: what happens for GPS satellites, Radio Sci., 39, RS5014, doi: 10.1029/ 2002RS002852.

Forte, B., S.M. Radicella and R. Leitinger (2004): Ionospheric radio wave scintillations observed during October 2003 storm at European auroral latitudes, in Beacon Satellite Symposium, 18-22 October 2004, Trieste, Italy.

Fremouw, E.J. (1980): Geometrical control of the ratio of intensity and phase scintillation indices, J. Atmos. Terr. Phys., 42, 775-782.

Fremouw, E.J., R.L. Leadabrand, R.C. Livingston, M.D. Cousins, C.L. RinO, B.C. FAIR and R.A. Long (1978): Early results from the DNA Wideband satellite esperiment-Complex signal analysis, Radio Sci., 13 (1), 167187.

Pi, X., B. Boulat, A.J. Mannucci, M. Reyes and D. StowERS (2001): Ionospheric scintillations measured using GPS receivers during the current solar maximum, in International Beacon Satellite Symposium, URSI 2001, Boston, MA.

RiNO, C.L. (1979): A power law phase screen model for ionospheric scintillation. 1. Weak Scatter, Radio Sci., 14 (6), 1135-1145.

RINO, C.L. and J. OwEN (1980): The structure of localized nighttime auroral zone scintillation enhancements, $J$. Geophys. Res., 85 (A6), 1941-1948.

VAn Dierendonck, A.J., J.A. Klobuchar and Q. HuA (1993): Ionospheric scintillation monitoring using commercial single frequency $\mathrm{C} / \mathrm{A}$ code receivers, in Proceedings of ION GPS-93, 1333, The Institute of Navigation, Arlington, VA.

(received June 22, 2007;

accepted March 3, 2008) 